\title{
XIII. Die Bestimmung der optischen Constanten eines Krystalles ans einem einzigen beliebigen Schnitte.
}

\author{
Von \\ C. Viola in Rom. \\ (Hierzu Taf. VIII, Fig. 3-6.)
}

Mit einem Totalreflectometer werden dic einzelnen Winkel der Totalreflexion zwischen einem einfachbrechenden Mittel von hohem Brechungsindex und einem doppeltbrechenden Mittel gemessen; ein Winkel der Totalreflexion heisse $i$, und sei $N_{0}$ der Brechungsindex des stark brechenden Mittels, z. B. in Bezug auf Iuft. lis frägt sich, was der Ausdruck

$$
N_{o} \sin i
$$

bedeute. Bei der Beantwortung dieser Frage werden wir zu Folgerungen kommen, welche unsere $\Lambda$ ufgabe lösen sollen.

Denken wir uns, dass $M M$ ('Taf. VIII, Fig. 3 oben) die horizontale Grenzebene zwischen dem unten liegenden cinfachbrechenden Mittel von dem Brechungsverhïltnisse $N_{o}$ und dem oben licgenden doppeltbrechenden Miltel darstellt. NIso ist die Zeichnungsebene zugleich die Einfallsebene. Unterhalb in der Fig. 3 wird die Grenzebene durch dic Zeichnungsebene dargestellt; also ist $J J$ die Spur der darauf senkrecht slehenden Einfallsebene. Der von unten kommende Lichtstrahl $S_{o}$ mit dem Einfallswinkel $i$ soll die Grenze der Totalreflexion angeben. Wir wollen zuerst kurz die Bedingungen dafür suchen, dass die Grenzc der Totalrellexion in der angenommenen Einfallsebene $J J$ unter dem Winkel $i$ stattlindet. Wir zeichnen mit dem Mittelpunkte $o$ und mit dem Halbmesser $o v_{o}$, der proportional zu der Lichtgeschwindigkeit $V_{o}$ im einfachbrechenden Mittel sein soll, eine llalbkugel (in der Verticalprojection $K$, Fig. 3), welche die Lichtwellenfläche für dieses einfachbrechende Miltel ist. In der IIorizontalprojection, Fig. 3 unten, wird diese Kugel durch den Kreis $K^{\prime}$ dargestellt. In demselben Massstabe zeichnet man auch die Wellenflïche für das doppeltbrechende Miltel; die Grösse der Normalen auf jeder Tangentialebene an dicser Wellenfläche ist 
die Lichtgeschwindigkeit der als Wellenebene aufgefassten Tangentialebene. Der Schnitt dieser Wellenfläche mit der Grenzebene der Totalreflexion soll die Curve $\Sigma$ sein (Taf. VIII, Fig. 3 unten). Wir begnügen uns damit, nur eine Curve des aus zwei Curven bestehenden Wellenflächenschnittes zu verzeichnen, denn was für die eine gesagt wird, gilt auch für die andere.

Ist also $i$ der Winkel der Totalreflexion, so wird die dem Strahle $S_{o}$ entsprechende Wellenebene $E_{0}$ eine solche Lage haben müssen, dass ihre Spur $E_{t} E_{t}$ auf der Grenzebene die Curve $\Sigma$ der Wellenfläche berührt. Durch den Berührungspunkt $s$ legt man die Tangentialebene $E$, Fig. 3, an die Wellenfläche, welche somit dieselbe Spur $E_{t} E_{t}$ haben wird wie die 'Tangentialebene $E_{0}$ an die Kugelfläche. So wird die Tangentialebene $E$ die Wellencbene in dem doppeltbrechenden Mittel darstellen, welche der Grenze der Totalreflexion entspricht; der lüngs der Grenzebene laufende Lichtstrahl für die Grenze der Totalreflexion wird os sein. Für dieselbe pflanzt sich die Lichtbewegung in der Richtung os fort, während die Wellenbewegung nach der zu der Wellenebene $E$ senkrecht stehenden und dem Brechungswinkel $e$ entsprechenden Richtung $o v$ vor sich geht. Bezeichnen wir dic Lichtgeschwindigkeit längs der Normalen $o v$ mit $V$, längs des Lichtstrahles os mit $S$, und führen wir noch die Geschwindigkeit der Lichtbewegung lïngs der Richtung ou cin, welche mit $U$ benannt sein mag. Sei ferner $\varepsilon$ der Winkel, den die lichtungen $o v$ und $o s$ mit einander einschliessen, Fig. $4^{1}$ ); $o u$ schliesse mil $o s$ den Winkel $\delta$ und mit $o v$ den Winkel $\varphi$ ein. Es ist nümlich $p=90^{\circ}-e$. 'Aus den Fig. 3, 4 entnehmen wir folgende Beziehungen :

oder

$$
\begin{gathered}
\overline{o v}=\overline{o u} \cos \varphi=\overline{o u} \sin e ; \overline{o v}_{o}=\overline{o u} \sin i, \\
\frac{1}{\bar{U}}=\frac{1}{V} \sin e=\frac{1}{V_{o}} \sin i .
\end{gathered}
$$

Ferner ergiebt sich noch

$$
\frac{1}{S}=\frac{1}{V} \cos \varepsilon=\frac{1}{V} \sin e \cos \delta \text {. }
$$

rühren wir jetzt die Brechungsindices ein.

$W$ sei die Lichtgeschwindigkeit in der Luft, so haben wir, wie bemerkt,

$$
\frac{I V}{V_{0}}=N_{o} \text {. }
$$

Ferner sei $N_{u}$ das Brechungsverhältniss für die Richtung ou und $N_{s}$ für dic Richtung des Strahles, so haben wir analog:

$$
\frac{W}{U}=N_{u} \text { und } \frac{W}{S}=N_{s} \text {. }
$$

Daher gehen unsere vorherigen Beziehungen in folgende über:

$$
N_{u}=N_{o} \sin i \text { und } N_{s}=N_{o} \cos \varepsilon \text {. }
$$

1) In Fig. 4, Taf. VIlI bedeuten die Punkte $s, u, v$ die Pole der Richtungen os, $o u, o v$. 
Wir künnen also unsere oben aufgeworfene Frage so beantworten:

Wenn man aus dem Winkel der Totalreflexion den Ausdruck $N_{o} \sin i$ bildet, so berechnet man das Brechungsvermögen der Lichtbewegung in der Richtung ou, welche sowohl in die Grenzebene als auch in die Einfallsebene füllt.

Aus der Beziehung

$$
\frac{1}{U}=\frac{1}{V_{0}} \sin i
$$

lïsst sich für alle Einfallsebenen die Lichtgeschwindigkeit für die in der Einfallsebene liegende Richtung berechnen. Tragen wir die so ermittelte Grösse $U$ auf den durch $o^{\prime}$ gehenden Radiusvector $J$ auf und führen wir dieselbe Operation für alle Richtungen aus, so werden wir eine gewisse Anzahl von Punkten bestimmen, durch welche eine Curve gehen wird; sie heisse die Curve $U$, welche direct aus den Messungen der Winkel der 'Totalreflexion hervorgeht. Dic Beziehung, welche die Curve $t$ mil der Schnittcurve $\Sigma$ der Wellenflïche hat, zeigt Fig. 3, Taf. VIII selbst. Die Curve $t^{T}$ geht nämlich durch die Fusspunkte der Normalen auf die Tangenten der Curve \. Ilat man daher die Curve $U$ durch die Messungen berechnet, so fillt man die Normalen an die Radienvectoren in allen Punkten dieser Curve, und man wird die Umhüllungscurve $\searrow$ ziehen künnen. Um so genauer wird eine solche Curve $\Sigma$ construirt werden künnen, je mehr 'langenten zu Gebote stehen werden. Wir sind also mit Hülfe der Winkel der 'Totalreflexion im Stande, den Schnitt der Grenzebene mit der Wellenflüche zu construiren, und zwar um so genauer, je zahlreichere Messungen vorliegen.

Wir berechnen gewühnlich anstatl der Curve $U$ ihre reciproke Curve $N_{u}$, welche aus der Beziehung

$$
N_{u}=N_{o} \sin i
$$

hervorgeht, wo $N_{o}$ constant ist.

Bezeichnet man die reciproke Curve $\Sigma$ mit $N_{s}$, so werden ihre Radienvectoren durch die Beziehung

berechnet.

$$
N_{s}=N_{o} \sin i \cos \delta
$$

Während die Tangenten an der Curve $\Sigma$ und die zu ihnen gefiilten Normalen die Fusspunkte bestimmen, durch welche die Curve $U$ geht, wird umgekehrt die reciproke Curve $N_{s}$ der Curve $\Sigma$ aus der reciproken Curve $N_{u}$ der Curve $U$ dadurch bestimmt, dass man die Tangenten an die Curve $N_{u}$ legt und die Fusspunkte der Normalen zu denselben construirt (Fig. $\ddot{6}$ ). Fs ist die Curve $U$ die Normalencurve der Curve $\Sigma$, und die Curve $N_{s}$ ist umgekehrt die Normalencurve der Curve $N_{u}$. Es ist alles reciprok, und wir künnen für unsere Betrachtungen beliebig von den Curven $\Sigma$ und $L$, oder von den Curven $N_{s}$ und $N_{u}$ ausgehen. Was bei dieser Reciprocität 
geändert wird, besteht nur in den Tangenten, denn diese stimmen in beiden Arten von Curven nicht mehr überein. Während die Curve $\mathbf{\Sigma}$ der Wellenfläche uns die Spur der Wellenebenen angiebt, thut das $N_{u}$ nicht mehr.

Indem wir also jetzt wissen, dass durch den Winkel der Totalreflexion $i$ und aus der Beziehung

$$
N_{u}=N_{o} \sin i
$$

die Curve $N_{u}$ berechnet wird, wollen wir fragen, was für Curven wir in dem Fernrohre des Refractometers beobachten. Man kann sagen, dass innerhalb eines kleinen Bereiches, wie es eben das Gesichtsfeld des Fernrohres ist, die Curve der Totalreflexion auf eine Ebene projicirt wird, welche zu einem mittleren reflectirten Lichtstrahle senkrecht steht. Sei ot die lichtung des zurückgeworfenen Lichtstrahles von der Grenzebene $M M$, Fig. 6, und gleichzeitig der optischen Axe des Fernrohres, $t d$ die Ebene, auf welcher das Bild der Grenze der. Totalreflexion sich projicirt, und der Abstand $o d=H$. Dann haben wir $\varrho=H \sin i$. Also ist der Radiusvector der im Gesichtsfelde erscheinenden Curve der Totareflexion proportional zu $\sin i$, genau wie der Radiusvector der Curve $N_{u}$. Die im Gesichtsfelde erscheinende Curve der Totalreflexion ist folglich mit der berechneten Curve $N_{u}$ identisch. $\Lambda$ nders verhält sich die Sache, wenn wir die ganze Curve der Totalreflexion auf ein Blatt Papier projiciren. Es sei z. B. $P P$ (Fig. 6) ein zur Grenzebene $M M$ paralleles Papier für die Projection der aus der Totalreflexion herkommenden Strahlen, und man wende Incidenzbeleuchtung mit allen Richtungen des Incidenzstrahles in der Grenzebene an. Der Radiusvector der auf $P P$ projicirten Curve wird offenbar $t c=r$ scin und steht in folgender Beziehung mit $i$ :

$$
r=h \text { tang } i \text {. }
$$

$\Lambda$ us dieser Untersuchung geht hervor, dass die Curve der Totalreflexion, wie sie durch das Fernrohr beobachtet wird, mit der Curve $N_{u}$ übereinslimmt, und wie sie auf ein Blatt Papier projicirt wird, von der Gurve $N_{u}$ verschieden ist.

1Fallt die Grenzebene der Totalreflexion mit einer optischen Hauptebene zusimmen, so stimmt die Curve $N_{u}$ mit dem Schnille der Indicesflüche überein, da die reciproke Curve $U$ in die Normalenfäche zu liegen kommt; fillt sie mit der Ebene der oplischen Axen zusammen, so schneiden sich die zwei Curven der Totalreflexion in den optischen Axen.

Dic Curve $U$ stimmt mit der Curve $\mathbf{\Sigma}$ nicht überein, ausgenommen in ganz speciellen Fällen. Fällt z. B. die Grenzebene mit einer optischen Hauptebene zusammen, so besteht die Curve $\Sigma$ aus einem Kreise und einer Ellipse. Die dem Kreise entsprechende Curve $U$ ist natürlich derselbe Kreis.

$\Lambda$ ber wenn die Curve $U$ mit der Curve $\Sigma$ nicht zusammenfällt, so giebt es doch gewisse Punkte, in denen die Curve $U$ die Curve $\Sigma$ berührt. Es handelt sich jetzt darum, zu untersuchen, wo die Berührungspunkte der 
beiden Curven liegen. Die Aufgabe könnte analytisch behandelt werden; wenn man aber den geometrischen Weg weiter einschlägt, gewinnt die Frage an Uebersichtlichkeit und der Beweis verliert nicht an Strenge.

Berührt sich die Curve $U$ mit der Curve $\boldsymbol{\Sigma}$ in einem Punkte, so fällt in diesem Punkte $u$ mit $s$, Fig. 3, Taf. VIII, zusammen. Tritt aber das ein, so haben beide Curven in dem speciellen Berührungspunkte eine gemeinsame Tangente, und überdies muss diese Tangente zum Radiusvector senkrecht stehen. Ist aber ferner der Radiusvector $o^{\prime} s$ resp. $o^{\prime} u$ zur Tangente beider Curven senkrecht, so stellt $o^{\prime} s$ resp. $o^{\prime} u$ ein Maximum oder ein Minimum dar.

Wir sind also jetzt zu folgendem Resultate gelangt: Die beiden Curven $U$ und $\Sigma$ müssen sich in solchen Punkten berühren, und $z$ war nur in solchen, wo Maxima und Minima der Radienvectoren zu Stande kommen. Solcher Maxima und Minima sind aber im Ganzen nur vier, wie die Erfahrung und die Theorie beweist; von diesen vier Maximis und Minimis gehören drei den Werthen $\mathfrak{a}, \mathfrak{b}$ und $\mathfrak{c}$ und der vierte einer dazwischen liegenden Grösse an, welche wir kurzweg mit $\mathfrak{b}^{\prime}$ bezeichnen wollen; alle vier gehüren naturgemüss der Wellenfläche an; somit werden mit llülfe der 'Totalreflexion immer vier Punkte der Wellenfläche direct bestimmt. Da $\mathfrak{a}$ und $\mathfrak{c}$ leicht und immer erkannt werden können, so liegt die nächste Aufgabe darin, die Stelle $\mathfrak{b}$ von der Stelle $\mathfrak{b}^{\prime}$ zu unterscheiden.

Diese Aufgabe wurde mit Benutzung eines einzigen beliebigen Schnittes des Krystalles für unlüsbar gehalten. Ich habe gezeigt ${ }^{1}$ ), dass, wenn man die Lage der Polarisationsebenen für die vier Strahlen auch nur annähernd in Bezug auf die Einfallsebene bestimmt, es durch eine kurze Rechnung leicht müglich ist, dic Strahlen $\mathfrak{b}$ und $\mathfrak{b}^{\prime}$ von einander zu unterscheiden. Es soll aber gezeigt werden, dass die Lösung dieser Aufgabe viel leichter und übersichtlicher sich gestalten kann, und dass jede Rechnung entbehrlich wird.

$\mathrm{Zu}$ diesem Zwecke fassen wir die Polarisationsrichtungen ins Auge.

Es sei in Erinnerung gebracht, dass die schwingende Bewegung des Lichtes in der Wellenebene erfolgt und zum Lichtstrahle senkrecht steht; sie muss in Folge dessen zu der in Fig. 4 Taf. VIII durch $v s$ dargestellten Ebene VS senkrecht stehen; also auch die Polarisationsebene muss zur Ebene VS senkrecht stehen. Dieses Merkmal führt uns sofort dazu, den Strahl $\mathfrak{b}^{\prime}$ zu erkennen. Dass für die Lage der Strahlen $\mathfrak{a}, \mathfrak{b}$ und $\mathfrak{c}$ die Punkte $u$ und $s$ zusammenfallen, wic immer die Polirisationsebenen auch liegen mögen, ist selbstverständlich, denn für die genannten drei Strahlen fällt $v$ mit $s$ zusammen. Da die Polarisalionsebenen dieser Strahlen in die optischen IIauptebenen fallen, so

1) C. Viola, Ueber Bestimmung der oplischen Constanten elc. Diese Zeitschr. 31, $40-48$. 
liegen sie zu der Grenzebene und der Einfallsebene der Totalreflexion im Allgemeinen geneigt. Nicht so für den Strahl $\mathfrak{b}^{\prime}$. Hier fällt $u$ mit $s$, aber nicht $v$ mit $s$ zusammen; also steht die Ebene $V S$ zur Grenzebene senkrecht, und somit fällt sie mit der Einfallsebene zusammen. Daher liegt die Polarisationscbene des Strahles $\mathfrak{b}^{\prime}$ zur Einfallsebene senkrecht.

Während also bei den drei Maximis und Minimis $\mathfrak{a}, \mathfrak{b}, \mathfrak{c}$ die Polarisationsebene gegen die Einfallsebene der Totalreflexion im Allgemeinen geneigt ist, ist sie dagegen für den vierten Strahl $\mathfrak{b}^{\prime}$, wo ebenfalls ein Maximum oder ein Minimum eintritt, immer senkrecht.

Nach diesem allgemeinen Satze müssen wir einige specielle Lagen der Grenzebene betrachten. Wir brauchen nicht die Fülle zu berücksichtigen, wo die Grenzebene durch eine optische Hauptaxe geht, oder mit einer oplischen Hauptebene zusammenfällt, denn in allen solchen Fällen sind die Grössen $\mathfrak{a}, \mathfrak{b}, \mathfrak{c}$ unzweideutig bestimmt. $\Lambda$ ber solche Fälle müssen näher geprüft werden, wo die Grenzebene nahe einer optischen Hauptrichtung, oder nahe einer optischen IIauptebene zu liegen kommt. Wir werden sehen, diss nur ein einziger Fall besondere Aufmerksamkeit verdient.

1. Wenn die Grenzebene einen sehr kleinen Winkel mit einer der drei optischen Hauptaxen einschliesst, so steht sie beinahe zu ciner Hauptebene senkrecht und ist gegen die anderen Hauptebenen geneigt. Für zwei der drei Strahlen $\mathfrak{a}, \mathfrak{b}, \mathfrak{c}$ wird daher die Polarisationsebene gegen die Einfallsebene geneigt sein, während für den dritten Strahl die Polarisationsebene eine sehr kleine Neigung mit der Einfallsebene bilden wird; für den vierlen Strahl $\mathfrak{b}^{\prime}$ steht die Polarisationsebene auf der Einfallsebene senkrecht, und somit bietet dieser Fall gar keine Schwierigkeit.

2. Die Grenzebene der Totalreflexion bildet mit einer der zwei optischen IIauptebenen $\mathfrak{b} \mathfrak{c}$ oder $\mathfrak{b} \mathfrak{a}$ einen sehr kleinen Winkel, also ist sie gegen die andere und gegen die dritte IIauptebene $\mathfrak{c a}$ nahezu normal. In diesem Falle wird die Polarisationsebene für einen der beiden Strahlen $\mathfrak{c}$ und $\mathfrak{a}$ nahezu senkrecht zur Einfallsebene stehen; aber für den Strahl $\mathfrak{b}$ wird die Polarisationsebene zu der Einfallsebene nahezu parallel sein. Da sie aber für den Strahl $\mathfrak{b}^{\prime}$ senkrecht steht, so werden auch in diesem Falle sofort $\mathfrak{b}$ und $\mathfrak{b}^{\prime}$ unterschieden werden können.

3. Die Grenzebene bildet mit der optischen Hauptebene $\mathfrak{c} \mathfrak{a}$, d. h. mit der Ebene der optischen Axen, einen sehr kleinen Winkel. Sie kommt also nahe den optischen Axen zu liegen. In diesem kritischen Falle hat der Strahl $\mathfrak{b}$ die Polarisationsebene sehr nahe senkrecht zur Einfallsebene. Für den Strahl $\mathfrak{b}^{\prime}$ liegt zwar die Polarisationsebene genau zur Einfallsebene senkrecht, aber da von einer grossen (ienauigkeit bei der Bestimmung der Polarisationsebene nicht die Rede sein kann, so bleiben die beiden Strahlen $\mathfrak{b}$ und $\mathfrak{b}^{\prime}$ auf diesem Wege unentschieden. Aber dieser kritische Fall kann 
nur in Betracht kommen, wenn die Grenzebene der Totalreflexion einen sehr kleinen Winkel mit der Ebene der optischen Axen einschliesst. Unter diesen Verhältnissen werden die innere und die äussere Grenzcurve der Totalreflexion sich in der Nähe der optischen Axen aneinander anschliessen. Man kann diher aus einem solchen Schnitte den Winkel der optischen Axen annähernd messen.

Ferner müssen die Strahlen $\mathfrak{b}$ und $\mathfrak{b}^{\prime}$ neben die Strahlen $\mathfrak{c}$ und $\mathfrak{a}$ fallen. Fällt also $\mathfrak{b}^{\prime}$ nahe an $\mathfrak{a}$ oder nahe an $\mathfrak{c}$, so sind die Brechungsverhältnisse der Strahlen $\mathfrak{b}^{\prime}$ und $\mathfrak{b}$, welche aus der Formel $N_{o} \sin i$ berechnet werden, sehr verschieden von einander. Berechnet man also den Winkel der optischen Axen mit der Tangentenformel, indem man für den mittleren Brechungsindex bald denjenigen des Strahles $\mathfrak{b}$, bald denjenigen des Strahles $\mathfrak{b}^{\prime}$ benutzt, und vergleicht dann diesen berechneten Winkel der optischen Axen mit dem gemessenen, so ist aus dem liesultate $\mathfrak{b}$ von $\mathfrak{b}^{\prime}$ sofort zu unterscheiden. Also crgiebt sich, dass der einzige, eine zweideutige Lösung hervorbringende kritische Fall der ist, wo die Grenzebene der Totalreflexion nahe der Ebene der optischen Axen kommt. Die Zweideutigkeit der Lüsung kann aber in den meisten Fällen verschwinden, wenn man durch die Grösse $\mathfrak{b}$ resp. $\mathfrak{b}^{\prime}$ den Winkel der optischen $\Lambda$ xen berechnet und mit dem gemessenen vergleicht.

Wir können in Folge dessen unserc Resultate so zusammenfassen:

Die aus dem Winkel der Totalreflexion sich ergebenden Maxima und Minima dienen dazu, unzweideutig die Grössen a, $\mathfrak{b}, \mathfrak{c}$ ohne Rechnung zu erhalten, indem man einfach von den zwei mittleren Werthen dasjenige Maximum oder Minimum ausscheidet, wo die Polarisationsebene zur Einfallsebene senkrecht steht.

Ist die Polarisationsebene für zwei nicht mit a oder $\mathfrak{c} z u-$ sammenfallende Strahlen der Maxima und Minima zur Einfallsebene nahezu senkrecht, so liegt die Grenzebene nahe der Ebene der optischen $A x e n$, und man hat den einzig möglichen kritischen Fall; die daraus entstehende $Z$ weideutigkeit kann dulch Messung des Winkels der optischen Axen beseitigt werden.

Zusatz bei der Corr. Nachdem obiger Aufsatz bereits dem Drucke übergeben war, kam mir eine interessante Publication über denselben Gegenstand von Cornu (Compt. rend. 133, 125 und Bull. soc. fr. d. min. 95, 7 f.) zu Gesicht. Unter dem Vorbehalt, bald auf die Frage zurückzukonmmen, erlaube ich mir hier nur darauf hinzuweisen, dass die von Cornu behandelte Aufgabe von mir schon vor mehreren Jahren vollstïndig gelöst worden ist (diese Zeitschr. 31, 40; siehe auch ebenda 31, $484 ; 32,113,305)$. 